\title{
First-in-Human Molecular Imaging of HER2 Expression in Breast Cancer Metastases Using the ${ }^{111}$ In-ABY-025 Affibody Molecule
}

\author{
Jens Sörensen ${ }^{1}$, Dan Sandberg ${ }^{1}$, Mattias Sandström ${ }^{1}$, Anders Wennborg ${ }^{2}$, Joachim Feldwisch ${ }^{2,3}$, Vladimir Tolmachev ${ }^{3}$, \\ Gunnar Åström ${ }^{4}$, Mark Lubberink ${ }^{1}$, Ulrike Garske-Román ${ }^{1}$, Jörgen Carlsson ${ }^{3}$, and Henrik Lindman ${ }^{4}$ \\ ${ }^{I}$ Nuclear Medicine and PET, Department of Radiology, Oncology, and Radiation Sciences, Uppsala University, Uppsala, Sweden; \\ ${ }^{2}$ Affibody AB, Solna, Sweden; ${ }^{3}$ Biomedical Radiation Sciences, Rudbeck Laboratory, Department of Radiology, Oncology, and \\ Radiation Sciences, Uppsala University, Uppsala, Sweden; and ${ }^{4}$ Oncology, Department of Radiology, Oncology, and Radiation \\ Sciences, Uppsala University, Uppsala, Sweden
}

\begin{abstract}
The expression status of human epidermal growth factor receptor type 2 (HER2) predicts the response of HER2-targeted therapy in breast cancer. ABY-025 is a small reengineered Affibody molecule targeting a unique epitope of the HER2 receptor, not occupied by current therapeutic agents. This study evaluated the distribution, safety, dosimetry, and efficacy of ${ }^{111}$ In-ABY-025 for determining the HER2 status in metastatic breast cancer. Methods: Seven patients with metastatic breast cancer and HER2-positive $(n=5)$ or -negative $(n=2)$ primary tumors received an intravenous injection of approximately $100 \mu \mathrm{g}(\sim 140 \mathrm{MBq})$ of ${ }^{111} \mathrm{In}-\mathrm{ABY}$-025. Planar $\mathrm{y}$-camera imaging was performed after $30 \mathrm{~min}$, followed by SPECT/CT after 4, 24, and $48 \mathrm{~h}$. Blood levels of radioactivity, antibodies, shed serum HER2, and toxicity markers were evaluated. Lesional HER2 status was verified by biopsies. The metastases were located by ${ }^{18} \mathrm{~F}-\mathrm{FDG}$ PET/CT $5 \mathrm{~d}$ before ${ }^{111}$ In-ABY-025 imaging. Results: Injection of ${ }^{111}$ In-ABY-025 yielded a mean effective dose of $0.15 \mathrm{mSv} / \mathrm{MBq}$ and was safe, well tolerated, and without drug-related adverse events. Fast blood clearance allowed high-contrast HER2 images within 4-24 h. No anti-ABY025 antibodies were observed. When metastatic uptake at $24 \mathrm{~h}$ was normalized to uptake at $4 \mathrm{~h}$, the ratio increased in HER2-positive metastases and decreased in negative ones $(P<0.05)$, with no overlap and confirmation by biopsies. In 1 patient, with HER2-positive primary tumor, ${ }^{111}$ In-ABY-025 imaging correctly suggested a HER2negative status of the metastases. The highest normal-tissue uptake was in the kidneys, followed by the liver and spleen. Conclusion: ${ }^{111}$ In-ABY-025 appears safe for use in humans and is a promising noninvasive tool for discriminating HER2 status in metastatic breast cancer, regardless of ongoing HER2-targeted antibody treatment.
\end{abstract}

Key Words: Affibody; breast cancer metastases; clinical study; HER2 receptor; ${ }^{111}$ In imaging

J Nucl Med 2014; 55:730-735

DOI: 10.2967/jnumed.113.131243

$\mathbf{T}$ oday, treatment of breast cancer is based on the biologic profile of the individual tumor. Knowledge of the human epidermal growth factor receptor type 2 (HER2) status is crucial to predict

Received Aug. 22, 2013; revision accepted Nov. 13, 2013.

For correspondence or reprints contact: Jens Sörensen, Nuclear Medicine and PET, Uppsala University Hospital, SE-75185, Uppsala, Sweden.

E-mail: jens.sorensen@ros.uu.se

Published online Mar. 24, 2014.

COPYRIGHT (c) 2014 by the Society of Nuclear Medicine and Molecular Imaging, Inc. the response of HER2-targeted therapy (1). Patients with breast cancer overexpressing HER2 have improved survival when treated with HER2-targeting agents such as trastuzumab, pertuzumab, and trastuzumab emtansine $(2-10)$.

The analysis of HER2 expression is usually based on a surgical specimen of the primary tumor or, in case of neoadjuvant therapy or inoperable disease, on a biopsy sample from the tumor (11). The pathologic analysis includes immunohistochemistry and in some cases fluorescence in situ hybridization (FISH). Therapy for patients with disseminated disease is often based on histopathologic classification of the primary tumor and not of the metastases. Disparities in HER2 expression of primary breast cancer and metastases have been reported. Metaanalysis of 26 studies including 2,520 patients revealed discordance in HER2 expression between the primary tumor and local lymph node metastases in the range of $2.4 \%-7.2 \%$ and discordance with distant metastases in the range of $6.9 \%-18.6 \%$, with an absolute variation for all studies in the range of $0 \%-40 \%$ (12). A recent symposium publication including 2,845 patients reported absolute variations in the same range (13). Another recent study on 182 patients, with $28 \%$ discordance, indicated that patients with loss of HER2 expression in metastases had shorter overall survival than patients with unchanged expression (14).

The biopsy procedure can be inconvenient or even harmful for the patient, demanding lesions of suitable size and carrying the risk of sampling errors. Heterogeneity of HER2 expression within lesions and differences in expression between lesions in the same patient further limit the use of biopsy for correct diagnosis. Thus, improved methods for determining the HER2 status in patients with metastatic breast cancer are needed to optimize treatment regimes. One approach is molecular imaging using a radiolabeled tracer targeting HER2.

Molecular imaging allows whole-body detection of aberrant gene expression (i.e., proteomic abnormalities). Radiolabeled trastuzumab has been clinically evaluated as a HER2-specific molecular imaging agent $(15,16)$. We used an imaging molecule with about 23 times smaller molecular weight, that is, Affibody molecules (Affibody $\mathrm{AB}$ ), and preclinical studies have shown promising results (17). Affibody molecules are small, approximately 6.5-kDa, imaging agents based on a nonimmunoglobulin scaffold. Target-specific Affibody molecules are selected from a library of several billion unique variants providing high-affinity binders to a variety of targets such as HER2 and have shown good imaging properties in xenograft models (17-23). The HER2-binding Affibody molecule used in this study binds with picomolar affinity to the extracellular 
domain 3 of the receptor, that is, to an epitope not overlapping with the epitopes for trastuzumab (domain 4) or pertuzumab (domain 2), thus permitting imaging during ongoing antibody therapy $(20,24,25)$.

Recently, clinical data using the first-generation HER2-binding Affibody molecule, ABY-002, demonstrated the feasibility of HER2 imaging with SPECT $\left({ }^{111} \mathrm{In}\right)$ and PET $\left({ }^{68} \mathrm{Ga}\right)$ in breast cancer patients (26). However, high liver uptake prevented visualization of liver metastases. ABY-025, used in the present clinical study, is a second-generation Affibody molecule with improved biochemical and biophysical characteristics, designed by protein engineering using an iterative approach of changing 11 amino acids (about $20 \%$ of the molecule) outside the HER2-binding region $(18,27)$.

In this first-in-human study with ${ }^{111}$ In-ABY-025 SPECT/CT, we evaluated safety and tolerability and explored uptake in tumor metastases and background uptake in normal organs. It was also of interest to study the ability of ${ }^{111}$ In-ABY-025 to discriminate between HER2positive and -negative metastases.

\section{MATERIALS AND METHODS}

\section{Patients}

Seven female patients (mean age, $61.3 \mathrm{y}$; range, 46-70 y) receiving treatment for recurrent metastatic breast cancer were enrolled into the study (Table 1). Five of the patients were diagnosed with HER2-positive primary tumors, and 2 had HER2-negative tumors and served as controls.

\section{Inclusion and Exclusion Criteria}

The protocol criteria for inclusion and exclusion are detailed in supplemental material (available at http://jnm.snmjournals.org). Briefly, patients with a diagnosis of metastatic breast cancer and a known HER2 classification of the primary tumor (HER2-positive: score of $3+$ using HercepTest [DAKO] or FISH-positive, or score of 2+ with HercepTest and FISH-positive; HER2-negative: score 0 or $1+$ using HercepTest, or score of $2+$ but FISH-negative) were potential participants. Ongoing treatment was not an exclusion criterion.

\section{Approvals}

The Swedish Medical Products Agency, the regional ethics committee in Uppsala, and the radiation protection ethics committee in Uppsala approved the study. Written informed consent was obtained from all participants. The study was registered as a clinical trial with the identifiers EudraCT 210-021078-12 and NCT01216033.

\section{Patient Characterization and Safety Assessment}

In accordance with the study protocol, all patients underwent physical examination at least $7 \mathrm{~d}$ before, immediately before, and $7 \mathrm{~d}$ after injection of ${ }^{111}$ In-ABY-025. The standard clinical chemistry of blood and urine was investigated according to the approved protocol. Possible adverse effects were investigated orally and with written patient questionnaires before and after ${ }^{111}$ In-ABY-025 injection (day 0) and 1, 7, 21, and $42 \mathrm{~d}$ later. Blood samples for determination of shed serum HER2 were taken immediately before injection, and the assays (ADIVA Centaur HER2/neu test; Siemens Healthcare Diagnostics) were thereafter performed at Laboratory Limbach, Heidelberg, Germany. The presence of anti-ABY-025 antibodies was determined in samples taken before, $21 \mathrm{~d}$ after, and $42 \mathrm{~d}$ after injections, using an ELISA developed by Affibody $\mathrm{AB}$ and performed at Clinical Chemistry and Pharmacology Laboratory at Uppsala University Hospital, Sweden.

\section{${ }^{18}$ F-FDG PET/CT and Other Clinical Imaging}

The metastatic status of patients was known before inclusion on the basis of conventional imaging. ${ }^{18} \mathrm{~F}-\mathrm{FDG}$ PET/CT imaging was performed $5 \mathrm{~d}$ before the ${ }^{111} \mathrm{In}$-ABY-025 administrations to identify the size and location of viable metastases in all patients. The patients fasted $6 \mathrm{~h}$ before ${ }^{18} \mathrm{~F}-\mathrm{FDG}$ injection. A scan (Discovery VCT; GE Healthcare) from head to thighs was performed $3 \mathrm{~h}$ after intravenous injection of 5 $\mathrm{MBq}$ of ${ }^{18}$ F-FDG. A low-dose CT scan (auto-mA, 20-80) without contrast enhancement was used for attenuation correction and anatomic localization. PET images were reconstructed using a clinical protocol supplied by the vendor, and all relevant corrections for quantitative imaging were applied. The acquired data were evaluated using Hermes Hybrid Viewer (Hermes Medical) and an Advance workstation (GE Healthcare). For each lesion detected by PET/CT, the maximum standardized uptake value was noted. The volume of each tumor lesion was calculated by a thresholding technique that included all voxels with at least $42 \%$ of the maximum standardized uptake value. Additionally, MR, ultrasound, or contrast-enhanced CT was applied when needed for biopsies and further patient management.

\section{${ }^{111}$ In-ABY-025 Imaging}

${ }^{111}$ In-ABY-025 was prepared essentially as described earlier (18). ABY-025 of good-manufacturing-practice grade was provided by Affibody $\mathrm{AB}$ in vials containing $100 \mu \mathrm{g}$. ABY-025 was labeled with ${ }^{111} \mathrm{In}$ at the Department of Nuclear Medicine, Uppsala University Hospital. Patients were not required to fast before injection. ${ }^{111} \mathrm{In}-\mathrm{ABY}-025$, about $100 \mu \mathrm{g}$,

TABLE 1

Patient Characteristics Before Injection with ${ }^{111}$ In-ABY-025

\begin{tabular}{|c|c|c|c|c|c|c|c|c|}
\hline $\begin{array}{l}\text { Patient } \\
\text { no. }\end{array}$ & $\begin{array}{l}\text { Age } \\
\text { (y) }\end{array}$ & $\begin{array}{l}\text { HER2 status } \\
\text { in primary tumor } \\
\text { (HercepTest) }\end{array}$ & $\begin{array}{l}\text { Primary } \\
\text { tumor } \\
\text { ER/PgR }\end{array}$ & $\begin{array}{l}\text { Pretreatment } \\
\text { serum HER2 } \\
\qquad(\mu \mathrm{g} / \mathrm{L})\end{array}$ & $\begin{array}{l}\text { Metastasis locations on } \\
{ }^{18} \text { F-FDG PET/CT }\end{array}$ & $\begin{array}{c}\text { Injected } \\
111 \text { In-ABY-025 } \\
\text { (MBq) }\end{array}$ & $\begin{array}{c}\text { On } \\
\text { trastuzumab } \\
\text { during } \\
\text { imaging }\end{array}$ & $\begin{array}{l}\text { Therapy } \\
\text { history* }\end{array}$ \\
\hline 1 & 69 & $3+$ & $-/-$ & 21 & Lymph node & 131 & Yes & $\mathrm{S}, \mathrm{R}, \mathrm{C}, \mathrm{T}, \mathrm{L}$ \\
\hline 2 & 57 & $3+$ & $+/-$ & 26 & Lymph node, liver, bone & 135 & Yes & $\mathrm{S}, \mathrm{R}, \mathrm{C}, \mathrm{E}, \mathrm{T}, \mathrm{L}$ \\
\hline 3 & 46 & $3+$ & $-1-$ & 56 & $\begin{array}{l}\text { Lymph node, bone, liver, } \\
\text { adrenal }\end{array}$ & 139 & Yes & $\mathrm{S}, \mathrm{R}, \mathrm{C}, \mathrm{T}, \mathrm{L}$ \\
\hline 4 & 70 & $3+$ & $+/-$ & 8.8 & $\begin{array}{l}\text { Lymph node, lung, bone, } \\
\text { brain, thyroid }\end{array}$ & 153 & Yes & $\mathrm{S}, \mathrm{R}, \mathrm{C}, \mathrm{E}, \mathrm{T}, \mathrm{L}$ \\
\hline 5 & 66 & $1+$ & $-1-$ & 16 & Lymph node, bone & 137 & No & S, R, C, \\
\hline 6 & 65 & 0 & $+/-$ & 12 & Bone, liver, lung & 139 & No & S, R, C, E \\
\hline 7 & 57 & $3+$ & $+/+$ & 23 & Lymph node, bone & 154 & Yes & $\mathrm{S}, \mathrm{C}, \mathrm{E}, \mathrm{T}, \mathrm{L}$ \\
\hline
\end{tabular}

*More information on therapy history is provided in the supplemental material.

$\mathrm{ER}$ = estrogen receptor; $\mathrm{PgR}$ = progesterone receptor; $\mathrm{S}=$ surgery; $\mathrm{R}=$ radiotherapy; $\mathrm{C}=$ chemotherapy; $\mathrm{E}=$ endocrine therapy; $\mathrm{T}=$ trastuzumab; $\mathrm{L}=$ lapatinib. 
TABLE 2

Uptake of ${ }^{111}$ In in Tumor-Free Areas of Organs with Highest Uptake on SPECT Images After Injection of ${ }^{111} \mathrm{In}-\mathrm{ABY}-025$

\begin{tabular}{lccc}
\hline \multicolumn{1}{c}{ Site } & $4 \mathrm{~h}$ & $24 \mathrm{~h}$ & $48 \mathrm{~h}$ \\
\hline Left kidney & $0.084 \pm 0.031$ & $0.103 \pm 0.030$ & $0.085 \pm 0.027$ \\
Right kidney & $0.090 \pm 0.034$ & $0.111 \pm 0.035$ & $0.093 \pm 0.032$ \\
Liver & $0.032 \pm 0.013$ & $0.025 \pm 0.010$ & $0.022 \pm 0.009$ \\
Spleen & $0.009 \pm 0.005$ & $0.007 \pm 0.005$ & $0.006 \pm 0.004$ \\
& & \\
&
\end{tabular}

TABLE 3

Level of ${ }^{111}$ In in Blood Samples After Injection of ${ }^{111} \ln -\mathrm{ABY}-025$

\begin{tabular}{lc}
\hline Time after injection & ${ }^{111}$ In level \\
\hline $10 \mathrm{~min}$ & $9.75 \times 10^{-3} \pm 3.90 \times 10^{-3}$ \\
\hline $30 \mathrm{~min}$ & $6.12 \times 10^{-3} \pm 2.15 \times 10^{-3}$ \\
$2 \mathrm{~h}$ & $3.46 \times 10^{-3} \pm 1.25 \times 10^{-3}$ \\
$6 \mathrm{~h}$ & $2.03 \times 10^{-3} \pm 0.70 \times 10^{-3}$ \\
$24 \mathrm{~h}$ & $0.63 \times 10^{-3} \pm 0.17 \times 10^{-3}$ \\
$48 \mathrm{~h}$ & $0.32 \times 10^{-3} \pm 0.06 \times 10^{-3}$ \\
$7 \mathrm{~d}$ & $0.10 \times 10^{-3} \pm 0.01 \times 10^{-3}$ \\
\hline
\end{tabular}

Data are percentage injected radioactivity per milliliter of blood (mean values and SD from all 7 patients).

with a mean activity of $142.6 \mathrm{MBq}$ (range, 131-154 MBq), was injected intravenously. At 4, 24, and $48 \mathrm{~h}$ after injection, whole-body planar scanning was performed followed by SPECT/CT (Infinia Hawkeye 4; GE Healthcare) over individually selected areas of special interest, defined by findings in an initial planar scan (anterior and posterior) after about $30 \mathrm{~min}$, as well as ${ }^{18} \mathrm{~F}-\mathrm{FDG}$ PET/CT. Low-dose CT scans without contrast enhancement were acquired for attenuation correction and anatomic correlation. SPECT data were reconstructed with CT-based attenuation correction into a $128 \times 128$ matrix using an iterative reconstruction algorithm.

\section{Blood Samples and Biopsies}

Blood samples were collected at 10 and $30 \mathrm{~min}$; at 2, 6, 24, and $48 \mathrm{~h}$; and at $7 \mathrm{~d}$ after injection to determine blood clearance kinetics. After the SPECT/CT results were known, optional biopsies were taken from suitable and clinically relevant lesions. In 2 patients (patients 1 and 2), metastases were surgically removed after the study. Biopsies were analyzed by immunohistochemistry (HercepTest) to verify the HER2 status.

\section{Statistical Analysis}

Quotients between the 24/4- or 48/4-h uptake of ${ }^{111} \mathrm{In}-\mathrm{ABY}-025$ were calculated, and 108 metastatic lesions larger than $1.5 \mathrm{~mL}$, as measured by ${ }^{18} \mathrm{~F}-\mathrm{FDG}$ PET/CT, were included in further analysis. The significance of differences between 4- and 24-h uptake values in HER2positive metastases was analyzed using nonparametric Kruskal-Wallis 1-way ANOVA. The significance of differences between quotient values and maximum standardized uptake value for HER2-positive and HER2negative metastases was analyzed using the nonparametric Mann-Whitney $U$ test. A 2 -sided $P$ value of less than 0.05 was considered significant.

\section{Supplemental Material}

Information on protocol inclusion and exclusion criteria, patient medical history, blood kinetics determination, ${ }^{111}$ In-ABY-025 imaging of normal-tissue uptake, dosimetry, the dual-time-point analysis, metastatic maximum standardized uptake value measurements, and biopsy data (including immunohistochemistry and evaluation criteria) are provided in the supplemental material.

\section{RESULTS}

\section{Safety Assessment}

The administration of ${ }^{111}$ In-ABY-025 was well tolerated. No clinically significant changes in laboratory evaluations or vital signs were recorded. No anti-ABY-025-specific antibodies could be detected in any of the patients 3 and $6 \mathrm{wk}$ after exposure.

\section{Pharmacokinetics, Biodistribution, and Dosimetry}

The blood kinetics of ${ }^{111} \mathrm{In}-\mathrm{ABY}-025$ and uptake in the kidney, liver, and spleen are presented in Tables 2 and 3 and in Supplemental Table 1. Clearance of ${ }^{111} \mathrm{In}$ from the blood was biphasic, with the first half-life being $2.9 \pm 0.5 \mathrm{~h}$ and the second half-life $27 \pm 5 \mathrm{~h}$. In normal organs, the highest uptake was observed in the kidney, followed by the

TABLE 4

Metastases Analyzed for ${ }^{111}$ In-ABY-025 Uptake Using SPECT/CT at Different Times After Injection

\begin{tabular}{|c|c|c|c|c|c|c|c|c|}
\hline \multirow{2}{*}{$\begin{array}{l}\text { Patient } \\
\text { no. }\end{array}$} & \multirow{2}{*}{$\begin{array}{c}\text { Metastases } \\
\text { detected with } \\
\text { 18F-FDG PET/CT }\end{array}$} & \multicolumn{3}{|c|}{$\begin{array}{c}\text { Metastases } \\
\text { with detectable } \\
{ }^{111} \text { In-ABY-025 uptake }\end{array}$} & \multirow[b]{2}{*}{ Biopsies } & \multirow{2}{*}{$\begin{array}{l}\text { HER2 status of biopsies } \\
\text { (immunohistochemistry) }\end{array}$} & \multirow{2}{*}{$\begin{array}{l}\text { HER2 status of } \\
\text { selected lesions from } \\
\text { imaging data using } \\
\text { 24/4-h quotient }\end{array}$} & \multirow{2}{*}{$\begin{array}{l}\text { HER2 status of } \\
\text { primary tumor } \\
\text { (Table 1) }\end{array}$} \\
\hline & & $4 \mathrm{~h}$ & $24 \mathrm{~h}$ & $48 \mathrm{~h}$ & & & & \\
\hline $1^{*}$ & 3 & 2 & 2 & 3 & 1 & Positive & Positive & Positive \\
\hline $2^{\star}$ & 17 & 10 & 13 & 13 & 1 & Positive & Positive & Positive \\
\hline $3^{\star}$ & 12 & 10 & 11 & 11 & 1 & Positive & Positive & Positive \\
\hline $7^{\star}$ & 21 & 16 & 21 & 19 & 1 & Positive & Positive & Positive \\
\hline $4^{\dagger}$ & 71 & 3 & 12 & 13 & 7 & Negative & Negative & Positive \\
\hline $5^{\dagger}$ & 79 & 37 & 37 & 36 & 1 & Negative & Negative & Negative \\
\hline $6^{\dagger}$ & 46 & 30 & 32 & 34 & 0 & No biopsy available & Negative & Negative \\
\hline
\end{tabular}

${ }^{*}$ No ${ }^{111} \mathrm{In}-\mathrm{ABY}-025$ uptake was visible on SPECT images of a few small lesions defined as metastases by ${ }^{18} \mathrm{~F}-\mathrm{FDG}$ PET/CT in patients $1,2,3$, and 7. Because of sensitivity limit of SPECT, it is not known whether these few were HER2-positive or HER2-negative.

${ }^{\dagger 111}$ In-ABY-025 uptake seen on ${ }^{18}$ F-FDG PET/CT-defined metastases from patients 4, 5 , and 6 gave low signals from large metastases and no signals from small metastases.

The 249 analyzed metastases were detected with ${ }^{18}$ F-FDG PET/CT 5 d before injection of ${ }^{111}$ In-ABY-025. 


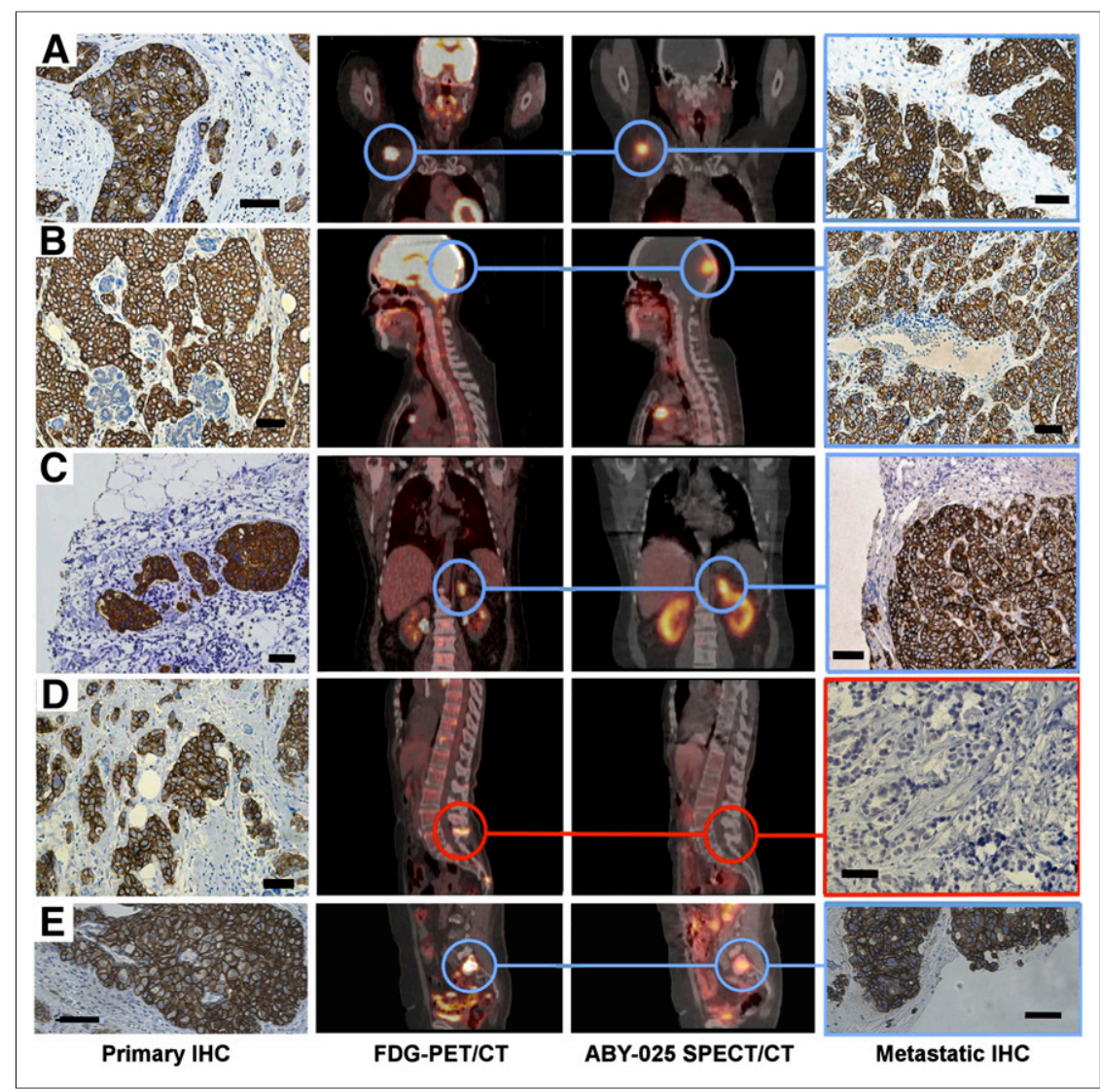

FIGURE 1. Examples of imaging of HER2 expression in breast cancer metastases using ${ }^{111}$ In-ABY025 SPECT/CT. Rows A, B, C, D, and E correspond to patients 1, 2, 3, 4, and 7, respectively. Left column shows positive immunohistochemistry $(\mathrm{IHC})$ staining of primary tumors, second column ${ }^{18} \mathrm{~F}$ FDG PET/CT scans, third column ${ }^{111}$ In-ABY-025-SPECT/CT scans, and fourth column immunohistochemistry staining of metastases. Blue circles indicate sites where biopsies were taken: patient 1 , lymph node metastasis; patient 2, brain metastasis; patient 3, adrenal metastasis; patient 4, bone metastasis; and patient 7 , bone metastases. Primary tumor from patient 4 showed immunohistochemistry $3+$ staining, and bone metastasis in spinal process showed high uptake of ${ }^{18} \mathrm{~F}-\mathrm{FDG}$. However, there was low (nearly no) uptake of ${ }^{111} \mathrm{In}-\mathrm{ABY}-025$ in metastasis, indicating HER2 negativity, and this was confirmed by immunohistochemistry-negative staining of biopsy sample. Patients 5 and 6 were HER2-negative in immunohistochemistry analysis of primary tumor, in ${ }^{111} \mathrm{In}$-ABY-025 scans of metastases, and in immunohistochemistry of metastases (immunohistochemistry only from patient 5; patient 6 refused biopsy). Bars in all immunohistochemistry images correspond to $50 \mu \mathrm{m}$.

liver and spleen. Uptake in the salivary glands and bowels was also visualized. No correlation was found between any organ uptake and shed serum HER2 $(8.8-56 \mu \mathrm{g} / \mathrm{L}$; the upper limit of normal is $15 \mu \mathrm{g} / \mathrm{L}$, Table 1). The normal organ receiving the highest radiation dose was the liver, followed by the kidneys and spleen, at $0.068 \pm 0.025,0.020 \pm$ 0.006 , and $0.005 \pm 0.002 \mathrm{mSv} / \mathrm{MBq}$, respectively. The effective radiation dose for the patients was $0.15 \pm 0.02 \mathrm{mSv} / \mathrm{MBq}$ (21 mSv per patient).

\section{Uptake in Metastases}

In the HER2-positive patients, large metastases $(>1 \mathrm{~cm})$ identified by ${ }^{18} \mathrm{~F}$-FDG PET/CT could be visualized with ${ }^{111} \mathrm{In}-\mathrm{ABY}-025$ in the first whole-body planar scan approximately $30 \mathrm{~min}$ after injection. Most metastases could be detected with high quality on images taken 4,24 , or $48 \mathrm{~h}$ after injection (Table 4). The mean uptake of ${ }^{111}$ In-ABY-025 in HER2-positive metastases was at least 1 order of magnitude higher than a calculated uptake for homogeneous distribution of radioactivity in the body at $24 \mathrm{~h}$ after injection. The HER2-positive metastases were visualized in different locations and tissues (examples for patients 1, 2, 3, and 7 are shown in Fig. 1). A brain metastasis of patient 2, not seen with ${ }^{18}$ F-FDG PET, was clearly visualized with ${ }^{111}$ In-ABY-025 and was confirmed as HER2positive by immunohistochemistry after surgical removal. The high HER2 expression in an adrenal gland metastasis in patient 3 was detected despite its proximity to the kidney. Bone metastases were clearly visualized in patients 2, 3, and 7. Patient 7 allowed biopsy of 1 bone metastasis, and HER2 positivity was confirmed by immunohistochemistry. Liver metastases were visualized in patient 2 (Supplemental Fig. 1). Biopsies from all 4 patients (1-3 and 7) taken from lesions defined with ${ }^{111}$ In-ABY-025 SPECT were HER2-stained and scored HER2 $3+$.

Patient 4 was included as a HER2-positive patient on the basis of an immunohistochemistry score of $3+$ for the primary tumor. However, ${ }^{111}$ In-ABY-025 SPECT showed low or no uptake in the ${ }^{18} \mathrm{~F}-\mathrm{FDG}$-defined lesions, and the HER2-negative status of biopsies from these lesions was confirmed by immunohistochemistry (Fig. 2 and supplemental material). Patients 5 and 6 had HER2-negative primary tumors; uptake of ${ }^{111}$ In-ABY-025 in their metastases was of low contrast, and the HER2-negative status was verified by immunohistochemistry on a biopsy sample from patient 5 (patient 6 refused biopsy).

\section{Discrimination Between Metastases with High and Low HER2 Expression}

The quantitative ${ }^{111}$ In-ABY-025 uptake in metastases classified as HER2-positive (patients 1, 2, 3, and 7) and HER2-negative (patients 4, 5, and 6) was different at both patient level and lesion level. The uptake in HER2-positive metastases increased between 4 and $24 \mathrm{~h}$, whereas the uptake in negative metastases generally was lower and decreased between 4 and $24 \mathrm{~h}$. HER2positive and HER2-negative lesions could be discriminated by calculating the decay-corrected 24/4-h uptake quotient. HER2-positive and HER2-negative metastases invariably showed a quotient greater than 1 and less than 1, respectively (Fig. 3). The difference between the groups classified as HER2-positive and HER2-negative was significant using a rank test $(P<0.05)$, with no overlap between the groups. The discriminatory capacity of this quotient was verified by immunohistochemistry on biopsied lesions.

Reevaluation of the original primary tumor tissue from patient 4 showed a heterogeneous HER2 expression, varying from 0 to $3+$ (immunohistochemistry examples are shown in Figures 2A, 2B, and $2 \mathrm{C}$ ), but the tumor was scored $3+$ since more than $10 \%$ of the cells were $3+$. The analysis of ${ }^{111}$ In-ABY025 uptake in the metastases indicated low or no HER2 expression (arrow in Fig. 3), that is, were HER2-negative. Immunohistochemistry analysis of the biopsy samples from patient 4 after ${ }^{111}$ In-ABY-025 imaging showed scores from 0 to $1+$ (immunohistochemistry examples are shown in Figs. 2F, 2G, and 2H). Thus, biopsy analysis of the metastases from patient 4 supported the use of 24/4-h quotients for discrimination. 


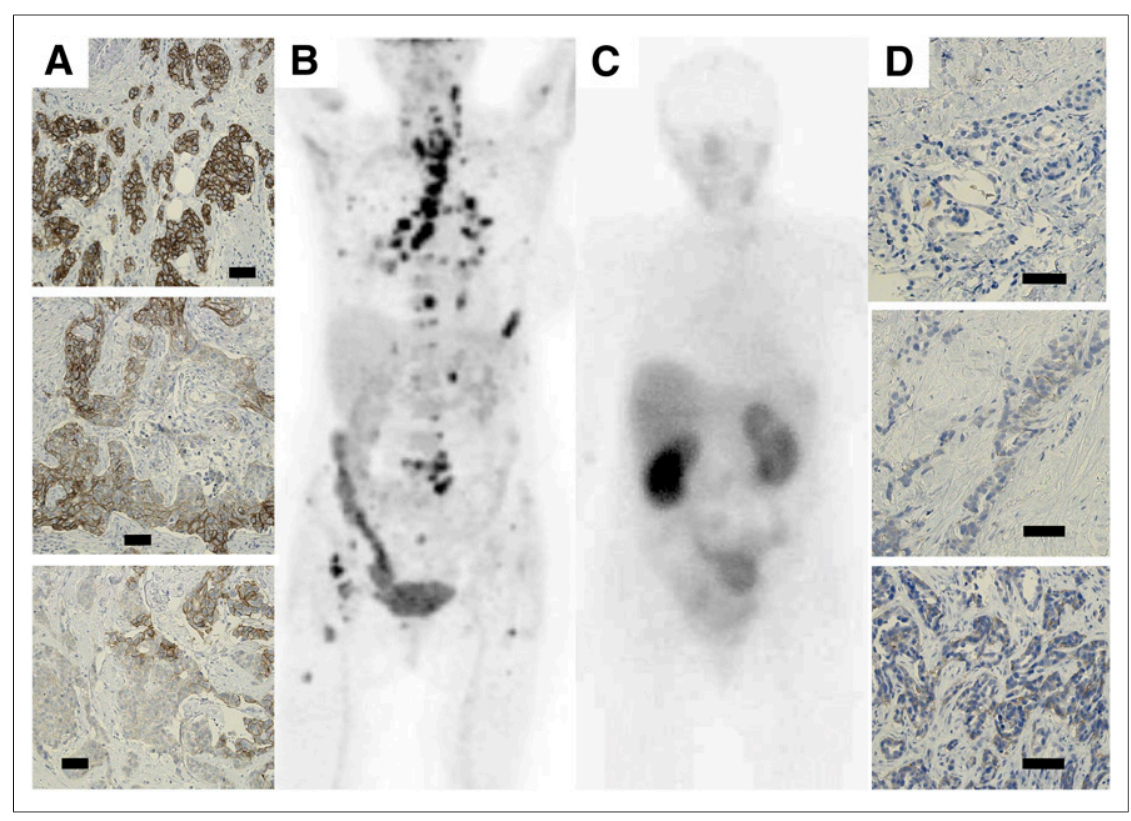

FIGURE 2. Patient 4 changed from HER2-positive primary tumor to HER2-negative metastases. Column A shows examples of variations in HER2 expression (immunohistochemistry) in primary tumor. More than $10 \%$ of tumor cells in primary tumor had strong circumferential HER2 staining of their entire cell membrane, and patient was therefore declared HER2-positive. B shows ${ }^{18} \mathrm{~F}-\mathrm{FDG}$ PET/CT scan 5 d before SPECT. In total, 71 metastases were detected (Table 4). C shows HER2 scan $4 \mathrm{~h}$ after injection of ${ }^{111} \mathrm{In}$-ABY-025. No HER2-expressing metastases could be detected in this scan, but 3 metastases with low HER2 expression could be detected in SPECT/CT sections after $4 \mathrm{~h}$ and more were detected after $24 \mathrm{~h}$ (Table 4). However 24/4-h quotients indicated that all these metastases were HER2-negative, as verified by immunohistochemistry analysis of biopsy samples. Column $D$ shows examples of immunohistochemistry analyses of 3 different metastases: thyroid metastasis that scored 0 (top), calcified thyroid metastasis that scored 0 (middle), and bone metastasis that scored 1+ (bottom). Bars in immunohistochemistry images correspond to $50 \mu \mathrm{m}$.

\section{DISCUSSION}

The results of this first-in-human exploratory study indicate that ${ }^{111}$ In-ABY-025 can be used as a whole-body-oriented, noninvasive agent to discriminate between HER2-positive and HER2-negative metastases. A single intravenous injection was well tolerated and safe and gave an effective patient dose of approximately $21 \mathrm{mSv}$.

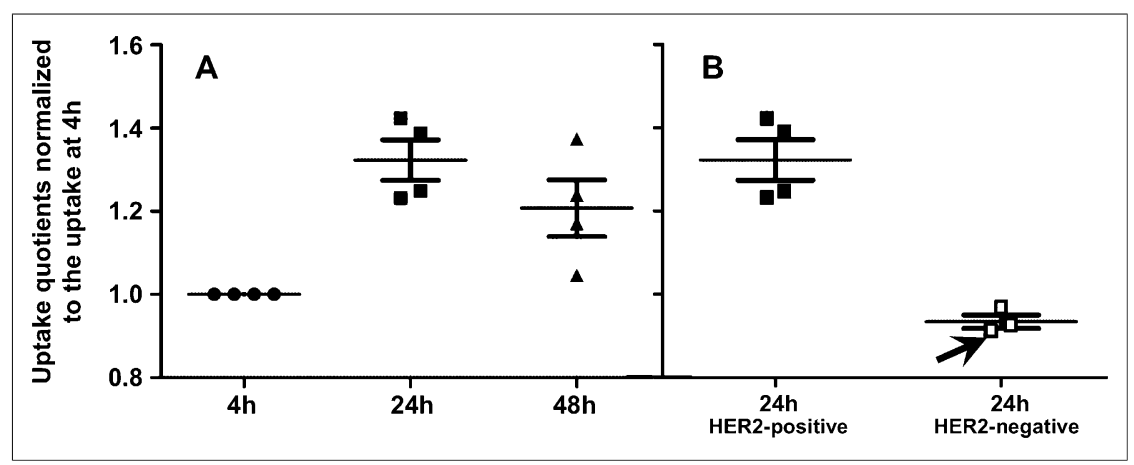

FIGURE 3. Plots of ${ }^{111} \mathrm{In}-\mathrm{ABY}-025$ uptake ratios in metastatic lesions defined by ${ }^{18} \mathrm{~F}-\mathrm{FDG}$ PET/CT. In total, 108 lesions were included in analysis. (A) Average ${ }^{111} \mathrm{In}$ uptake at 24 and $48 \mathrm{~h}$ calculated for each patient with HER2-positive metastases and normalized to uptake at $4 \mathrm{~h}$ after injection. ${ }^{111}$ In uptake increased significantly in all lesions from $4 \mathrm{~h}(\bullet)$ to $24 \mathrm{~h}(\boldsymbol{\square})$ and remained increased at $48 \mathrm{~h}(\mathbf{\Delta})$ after injection (Kruskal-Wallis ANOVA, $P=0.01$ ). (B) Average ${ }^{111} \mathrm{In}$ uptake

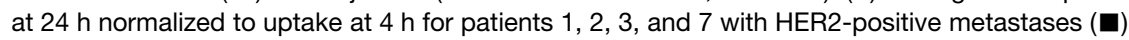
and for patients 4,5 , and 6 with HER2-negative metastases ( $\square$ ). There was no overlap between values for HER2-positive and HER2-negative metastases, and difference was significant (MannWhitney, $P<0.05)$. Mean values and SE estimates are given. Arrow in $B$ indicates data for patient 4, that is, patient who had HER2-positive primary tumor but HER2-negative metastases.
No drug-related adverse events or antiABY-025 antibodies were observed.

The rapid clearance of ${ }^{111}$ In from blood and normal organs allowed HER2 imaging of large $(>1 \mathrm{~cm})$ metastases $30 \mathrm{~min}$ after injection and gave images with good contrast after 4,24 , and $48 \mathrm{~h}$. The levels of shed serum-HER2 did not appear to affect normal-organ uptake or blood kinetics.

The high uptake of ${ }^{111} \mathrm{In}$-ABY-025 in metastases from patients 1, 2, 3, and 7 provided excellent HER2 visualization throughout the body. Immunohistochemistry analysis of biopsies confirmed the overexpression of HER2. Thus, imaging with ${ }^{111} \mathrm{In}-\mathrm{ABY}-025$ can identify HER2-positive metastases. However, large lesions could also be visualized in HER2-negative patients 5 and 6, although with weak signals (Table 4). This can be explained by the fact that tumors with HercepTest scores 0 and $1+$ may have up to 15,000-25,000 and 80,000-110,000 HER2 receptors per cell, respectively (28). Thus, SPECT-based imaging appears sensitive enough to visualize even low HER2 expression. Preclinical studies have shown that discrimination between tumors with high and low levels of HER2 expression is possible, either using Affibody molecules with low specific radioactivity (23) or using the fast clearance of radioactivity from tumors with low HER2 expression (29). In the present study, the decay-corrected 24/4-h uptake ratio was used to determine the HER2 status at both patient and lesion level. The average ${ }^{111} \mathrm{In}$-ABY-025 uptake increased significantly in all lesions from 4 to $24 \mathrm{~h}$ and remained increased at $48 \mathrm{~h}$ for HER2-positive patients 1, 2, 3, and 7. In contrast, lesions from HER2-negative patients 5 and 6 showed decreased ${ }^{111}$ In-ABY-025 uptake from 4 to $24 \mathrm{~h}$. The 24/4-h uptake ratios were invariably greater than 1 for HER2-positive lesions and less than 1 for HER2-negative lesions. The validity of this approach was supported by data from patient 4 (primary tumor HercepTest score, 3+) demonstrating low ${ }^{111} \mathrm{In}-\mathrm{ABY}$ 025 uptake in the metastases. Analysis of all lesions from this patient showed 24/4-h quotients of less than 1, typical for low HER2 expression, and immunohistochemistry analysis of biopsies confirmed low HER2 expression with HercepTest scores of 0 or $1+$.

The 24/4-h quotient method requires a 2-d protocol. A single-time-point protocol using SPECT/CT appears to be feasible (supplemental material) but may optimally require PET technique since the sensitivity and absolute quantification are better than for SPECT. Preclinical studies have demonstrated that Affibody molecules can be labeled with positron emitters such as ${ }^{68} \mathrm{Ga}$ and ${ }^{18} \mathrm{~F}$ with preserved HER2-targeting capacity $(22,30-32)$. 
Interestingly, the use of ${ }^{111}$ In-ABY-025 allowed HER2 imaging of known liver metastases in patient 2 . This is an improvement since liver metastases could not be visualized using the first-generation anti-HER2 Affibody molecule, ABY-002 (Supplemental Fig. 1) (26). ABY-025, used in the current study, has been obtained by protein engineering to increase hydrophilicity, increase thermal stability, increase production characteristics (27), and, as shown in animal experiments, lower liver uptake (18). The present study suggests that the changes engineered into ABY-025 provide clinical utility. The physiologic liver uptake varied between the patients. Patient 2 had the lowest physiologic liver uptake and fasted before administration of ${ }^{111} \mathrm{In}$-ABY-025, whereas patients eating before administration had higher physiologic liver uptake.

Radiolabeled trastuzumab has been evaluated earlier for HER2 imaging $(15,16)$, and image quality has been reported to be optimal 4-5 d after injection (15), compared with 4-24 h using ${ }^{111}$ In-ABY-025. The limited number of reported clinical studies does not permit a detailed comparison of sensitivity and specificity of radiolabeled trastuzumab versus ${ }^{111}$ In-ABY-025 in the clinical setting. The unique binding epitope of ABY-025, which is different from the epitopes of either trastuzumab or pertuzumab (24), allowed imaging during trastuzumab treatment.

\section{CONCLUSION}

Our findings indicate that imaging of breast cancer metastases with ${ }^{111} \mathrm{In}-\mathrm{ABY}-025$ is feasible and might be valuable for selection of patients who may, or may not, benefit from HER2-targeted therapies, hence improving treatment utility and cost-effectiveness.

\section{DISCLOSURE}

The costs of publication of this article were defrayed in part by the payment of page charges. Therefore, and solely to indicate this fact, this article is hereby marked "advertisement" in accordance with 18 USC section 1734 . The Swedish Cancer Society provided financial support (contracts 110565 and 120415). No other potential conflict of interest relevant to this article was reported.

\section{ACKNOWLEDGMENTS}

We thank the staff of the Department of Nuclear Medicine, Uppsala University Hospital, and Research Nurse Jessica Barrefjord, Department of Oncology, Radiology, and Radiation Sciences, Uppsala University, Sweden, for administration and patient care.

\section{REFERENCES}

1. Goldhirsch A, Wood WC, Coates AS, et al. Strategies for subtypes: dealing with the diversity of breast cancer-highlights of the St. Gallen International Expert Consensus on the Primary Therapy of Early Breast Cancer 2011. Ann Oncol. 2011;22:1736-1747.

2. Awada A, Bozovic-Spasojevic I, Chow L. New therapies in HER2-positive breast cancer: a major step towards a cure of the disease? Cancer Treat Rev. 2012;38:494-504.

3. Burstein HJ. Patients with anti-HER2 responsive disease: definition and adjuvant therapies [abstract]. Breast. 2011;20(suppl 3):S132-S134.

4. Cortés J, Fumoleau P, Bianchi GV, et al. Pertuzumab monotherapy after trastuzumab-based treatment and subsequent reintroduction of trastuzumab: activity and tolerability in patients with advanced human epidermal growth factor receptor 2-positive breast cancer. J Clin Oncol. 2012;30:1594-1600.

5. Higgins MJ, Baselga J. Targeted therapies for breast cancer. J Clin Invest. 2011;121:3797-3803.

6. Murphy CG, Morris PG. Recent advances in novel targeted therapies for HER2positive breast cancer. Anticancer Drugs. 2012;23:765-776.

7. Perez EA, Romond EH, Suman VJ, et al. Four-year follow-up of trastuzumab plus adjuvant chemotherapy for operable human epidermal growth factor receptor 2-positive breast cancer: joint analysis of data from NCCTG N9831 and NSABP B-31. J Clin Oncol. 2011;29:3366-3373.

8. Perez EA, Suman VJ, Davidson NE, et al. Sequential versus concurrent trastuzumab in adjuvant chemotherapy for breast cancer. J Clin Oncol. 2011;29:4491-4497.

9. Slamon DJ, Leyland-Jones B, Shak S, et al. Use of chemotherapy plus a monoclonal antibody against HER2 for metastatic breast cancer that overexpresses HER2. N Engl J Med. 2001;344:783-792.

10. Verma S, Miles D, Gianni L, et al. Trastuzumab emtansine for HER2-positive advanced breast cancer. $N$ Engl J Med. 2012;367:1783-1791.

11. Wolff AC, Hammond ME, Schwartz JN, et al. American Society of Clinical Oncology/ College of American Pathologists guideline recommendations for human epidermal growth factor receptor 2 testing in breast cancer. J Clin Oncol. 2007;25:118-145.

12. Houssami N, Macaskill P, Balleine RL, Bilous M, Pegram MD. HER2 discordance between primary breast cancer and its paired metastasis: tumor biology or test artefact? Insights through meta-analysis. Breast Cancer Res Treat. 2011;129:659-674.

13. Foukakis T, Astrom G, Lindstrom L, Hatschek T, Bergh J. When to order a biopsy to characterise a metastatic relapse in breast cancer. Ann Oncol. 2012;23(suppl 10): x349-x353.

14. Niikura N, Liu J, Hayashi N, et al. Loss of human epidermal growth factor receptor 2 (HER2) expression in metastatic sites of HER2-overexpressing primary breast tumors. J Clin Oncol. 2012;30:593-599.

15. Dijkers EC, Oude Munnink TH, Kosterink JG, et al. Biodistribution of ${ }^{89} \mathrm{Zr}$ trastuzumab and PET imaging of HER2-positive lesions in patients with metastatic breast cancer. Clin Pharmacol Ther. 2010;87:586-592.

16. Perik PJ, Lub-De Hooge MN, Gietema JA, et al. Indium-111-labeled trastuzumab scintigraphy in patients with human epidermal growth factor receptor 2-positive metastatic breast cancer. J Clin Oncol. 2006;24:2276-2282.

17. Löfblom J, Feldwisch J, Tolmachev V, Carlsson J, Stahl S, Frejd FY. Affibody molecules: engineered proteins for therapeutic, diagnostic and biotechnological applications. FEBS Lett. 2010;584:2670-2680.

18. Ahlgren S, Orlova A, Wallberg H, et al. Targeting of HER2-expressing tumors using ${ }^{111}$ In-ABY-025, a second-generation Affibody molecule with a fundamentally reengineered scaffold. $J$ Nucl Med. 2010;51:1131-1138.

19. Orlova A, Magnusson M, Eriksson TL, et al. Tumor imaging using a picomolar affinity HER2 binding affibody molecule. Cancer Res. 2006;66:4339-4348.

20. Orlova A, Tolmachev V, Pehrson R, et al. Synthetic affibody molecules: a novel class of affinity ligands for molecular imaging of HER2-expressing malignant tumors. Cancer Res. 2007;67:2178-2186.

21. Tolmachev V. Imaging of HER-2 overexpression in tumors for guiding therapy. Curr Pharm Des. 2008;14:2999-3019.

22. Tolmachev V, Velikyan I, Sandstrom M, Orlova AA. HER2-binding Affibody molecule labelled with ${ }^{68} \mathrm{Ga}$ for PET imaging: direct in vivo comparison with the ${ }^{111}$ In-labelled analogue. Eur J Nucl Med Mol Imaging. 2010;37:1356-1367.

23. Tolmachev V, Wallberg H, Sandstrom M, Hansson M, Wennborg A, Orlova A. Optimal specific radioactivity of anti-HER2 Affibody molecules enables discrimination between xenografts with high and low HER2 expression levels. Eur J Nucl Med Mol Imaging. 2011;38:531-539.

24. Eigenbrot C, Ultsch M, Dubnovitsky A, Abrahmsen L, Hard T. Structural basis for high-affinity HER2 receptor binding by an engineered protein. Proc Natl Acad Sci USA. 2010;107:15039-15044.

25. Kramer-Marek G, Gijsen M, Kiesewetter DO, et al. Potential of PET to predict the response to trastuzumab treatment in an ErbB2-positive human xenograft tumor model. J Nucl Med. 2012;53:629-637.

26. Baum RP, Prasad V, Muller D, et al. Molecular imaging of HER2-expressing malignant tumors in breast cancer patients using synthetic ${ }^{111} \mathrm{In}$ - or ${ }^{68} \mathrm{Ga}$-labeled Affibody molecules. J Nucl Med. 2010;51:892-897.

27. Feldwisch J, Tolmachev V, Lendel C, et al. Design of an optimized scaffold for affibody molecules. J Mol Biol. 2010;398:232-247.

28. Ross JS, Fletcher JA, Bloom KJ, et al. Targeted therapy in breast cancer: the HER-2/neu gene and protein. Mol Cell Proteomics. 2004;3:379-398.

29. Tolmachev V, Tran TA, Rosik D, Sjoberg A, Abrahmsen L, Orlova A. Tumor targeting using Affibody molecules: interplay of affinity, target expression level, and binding site composition. J Nucl Med. 2012;53:953-960.

30. Cheng Z, De Jesus OP, Namavari M, et al. Small-animal PET imaging of human epidermal growth factor receptor type 2 expression with site-specific ${ }^{18} \mathrm{~F}$-labeled protein scaffold molecules. J Nucl Med. 2008;49:804-813.

31. Heskamp S, Laverman P, Rosik D, et al. Imaging of human epidermal growth factor receptor type 2 expression with ${ }^{18} \mathrm{~F}$-labeled Affibody molecule ZHER2:2395 in a mouse model for ovarian cancer. $J$ Nucl Med. 2012;53:146-153.

32. Kramer-Marek G, Kiesewetter DO, Martiniova L, Jagoda E, Lee SB, Capala J. $\left[{ }^{18}\right.$ F]FBEM-Z(HER2:342)-Affibody molecule: a new molecular tracer for in vivo monitoring of HER2 expression by positron emission tomography. Eur J Nucl Med Mol Imaging. 2008;35:1008-1018. 